\title{
Schreiben des Herrn Prof. C. Fearnley an den Herausgeber.
}

Herr Observator Geelmuyden theilt mir soeben mit, dass ein von uns viermal beobachteter Stern 9. Grösse B.D. $11^{\mathrm{b}} 12^{\mathrm{m}} 27^{\mathrm{s}}+66^{0} 37^{\prime} . \overline{0}(185 \overline{)})$ eine s tarke Eigen bewegung hat. Dieses Resultat gelit schon aus den Christiania-Beobachtungen selbst mit der grössten Deutlichkeit hervor, wird aber ausserdem durch eine Beob. achtung von Argelander vollkommen bestätigt.

Die beobachteten auf den für $187 \overline{0} 0$ geltenden Aequator und Frühlingspuukt bezogenen Oerter waren: 1843 März $311^{\mathrm{h}} 13^{\mathrm{m}} 46^{\mathrm{s}} 83+6^{\mathrm{o}} 31^{\prime} 18^{\prime \prime} 3$ A.-Oe. 11677 ; A.R. um $-0^{\mathrm{s}} 15$ corr. nach Arg. i. Viert. Schr. VIII.

\begin{tabular}{|c|c|c|}
\hline 1870 April 19 & 33.17 & 23.2 Chr. Z. 9. Kr. Ost. \\
\hline 1871 April $\overline{0}$ & 32.60 & Kr. Wes \\
\hline 1873März $3 \mathrm{~L}$ & 31.48 & $\mathrm{Kr} . \mathrm{Os}$ \\
\hline 1873 April 16 & 31.50 & "136.Kr. Wes \\
\hline
\end{tabular}

Das Mittel aus den Christianiabeobachtungen $11^{\mathrm{h}} 13^{\mathrm{m} 32^{\mathrm{s}} 19}+66^{\circ} 31^{\prime} 24^{\prime \prime} 3$

giebt mit dem Argelanderschen Orte verglichen für die Eigenbewegung in $28.8 \overline{5}$ Jahren

$$
-14 \mathrm{~s} 64 \text { und }+6.0
$$

also dio jährliche Eigenbewegung

$$
\Delta r=-0 \mathrm{0} 0074 \quad \Delta^{\delta}=+0^{\prime \prime} 208
$$

oder im Bogen des grüssten Kreises

$$
3 " 04 \text {. }
$$

Mit Berücksichtigung dieser Eigenbewegung erhält man den mittleren Ort fin 1875.0: $11^{\mathrm{h}} 13^{\mathrm{m}} 30^{\mathrm{s}} 68+66^{0} 31^{\prime} 24^{\prime \prime} 95$

mit folgenden im Sinne Beob.-Rechn. übrigbleibenden Differenzen :

in A.R. $0^{\mathrm{s}} 00+0^{\mathrm{s}} 11+0^{\mathrm{8}} 02-0^{\mathrm{s}} 09-0^{\mathrm{s}} 05$ in Decl. $0^{\prime \prime} 0-0^{\prime \prime} 7+1^{\prime \prime} 2+1^{\prime \prime} \overline{0}-2^{\prime \prime} 0$

Der Stern ist in keinern andern uns zugänglichen Catalog zu finden.

Aus den hiesigen Beobachtungen allein würde man $\Delta^{x}=-0^{\mathrm{B}} 559$ und fur $187 \mathrm{j} .0$

$\alpha=11^{\mathrm{h}} 13^{\mathrm{m}} 30^{\mathrm{s}} 54 \quad 30^{\mathrm{s}} 50 \quad 30^{\mathrm{s}} 49 \quad 30^{\mathrm{s}} 54$ erhalten.

Die von mir geschätzte Grösse war 9mj $99^{\mathrm{m}} 0 \quad 9^{\mathrm{m} 0} \quad 9^{\mathrm{m}} 2$

Die erste Schätzung ist aber wie die benachbarten Sterne der Zone 9 deutlich zeigen um etwa eine halbe Grössenklasse zu schwach. Argelander notirt ebenfalls 9 .

Von dem Apex der Sonnenbewegung ist dieser Stern etra $60^{\circ}$ entfernt. Seine Lage ist also der parallaktischen Abspiegelung dieser Bewegung selir günstig. In der That entfernt sich nun auch der Stern vom Apex in eine Richtung, die nur wenig von der parallaktischen Bewegungsrichtung abweicht. Die beobachtete Ortsveränderung des Sterns dürfte daher wohl grösstentheils der Sonnenbewegung zuzuschreiben sein und daher zu der Hoffuung berechtigen bei diesem Storn eine verhältnissmässig grosse Parallaxe constatiren zu können.

Christiania Sternwarte 1878 Febr. 21

$$
\text { C. Fearnley. }
$$

\section{Anzeige.}

Soweit der Vorrath reicht, ist dureh jede Buchhandlung zu bezieben:

Zeitsehrift für popul. Mittheilungen aus dem Gebiete der Astronomie und rerwandter Wissenschaften, herausgegeben ron Professor Peters in Kicl.

3 Bäude. Preis 6 Mark. (früherer Preis $9 \mathrm{Mlk}$.)

Leipzig, Aprii 1878.

Wilh. Mauke.

\section{Inhalt:}

Zu Nr. 2191. Th. v. Oppolzer. Einige Bemerkungen über die Bahnbestimmung aus drei Orten. 97. - Fr. Sikzuab. Resulta:e aus Beobachtungen veränderlicher Sterne im Jahre 1877. 103. - Entdeckung eines Planeten. III.

Zu Nr. 2192. Winnecke. Beobachtungen des Mondes und der Mondsterne im Jahre 1877 auf der prov. Universitätssternwarte zu Strassburg. I 13 . $H$. Dembotuski. Beobachtungen von Doppelsternen. 121. - C. Fcarnlcy. Schreiben an den Heransgeber. 127. - W. Mauke. Anzeige. 127. 